\title{
Unexciting classical backgrounds
}

\author{
Tanmay Vachaspatiఠ \\ Physics Department, Arizona State University, Tempe, Arizona 85287, USA
}

(Received 12 January 2022; accepted 17 February 2022; published 8 March 2022)

\begin{abstract}
Quantum fields in time-dependent backgrounds generally lead to particle production. Here we consider "unexciting" backgrounds for which the net particle production vanishes. We start by considering the simple harmonic oscillator and explicitly construct all unexciting time-dependent frequencies. This allows us to construct homogeneous backgrounds in field theory for which there is no particle production in any given mode, though we are able to show that there are no homogeneous backgrounds for which the particle production vanishes in every mode. We then construct general inhomogeneous unexciting field theory backgrounds. The set of all unexciting field theory backgrounds will be further restricted by the choice of physical interactions and this leads to an interesting open problem.
\end{abstract}

DOI: 10.1103/PhysRevD.105.056008

\section{INTRODUCTION}

There has been considerable effort to study quantum radiation in time-dependent classical backgrounds (e.g., [1]). Landmark examples include Schwinger particle production [2] and Hawking radiation [3]. In the latter, the time dependence of the metric during gravitational collapse produces particles, while Schwinger particle production can be thought of as due to the time dependence of the electromagnetic vector gauge potential.

The present work is motivated by the Schwinger process for non-Abelian gauge fields recently discussed in Ref. [4] where a homogeneous non-Abelian electric field of a certain "color" produces (massless) gauge radiation of other colors. This process appears to be quite general, so one might expect a similar process to occur even if the background electric field is not uniform, for example if the color electric field is confined into flux tubes, as is widely believed to occur in QCD. However, QCD electric flux tubes should not produce quantum excitations if they are to be stable and confining. This motivates the general question-can we find nontrivial space- and time-dependent backgrounds in which particle production does not occur?

An example of an unexciting electric field configuration is already known in massless QED in $1+1$ dimensions $[5,6]$. One considers a capacitor consisting of external charges $+Q$ and $-Q$ separated by a distance $L$. The system can be solved completely since bosonization yields a scalar

Published by the American Physical Society under the terms of the Creative Commons Attribution 4.0 International license. Further distribution of this work must maintain attribution to the author(s) and the published article's title, journal citation, and DOI. Funded by SCOAP ${ }^{3}$. plus gauge field theory with only bilinear couplings. The unexciting electric field background takes the form [5],

$$
\begin{aligned}
F_{01}= & Q(\Theta(x+L / 2)-\Theta(x-L / 2)) \\
& +g(f(x+L / 2)-f(x-L / 2))
\end{aligned}
$$

where $g$ is the coupling constant in the model, and

$$
f(x)=-\frac{Q}{2 g} \operatorname{sgn}(x)\left(1-e^{-g|x|}\right)
$$

A sketch of the unexciting electric field is shown as the dashed curve in Fig. 1. Note, though, that the unexciting background is not purely an electric field as it also consists of a condensate of fermion bound states. These bound states are described after bosonization by a scalar field, $\phi$, that acquires a nontrivial profile,

$$
\phi(x)=f(x+L / 2)-f(x-L / 2) .
$$

Unexciting backgrounds may have practical utility as well. We can imagine situations where a quantum system is in its ground state in a certain background (e.g., a magnetic field), and we would like to change the background to a final configuration while the quantum system is finally in its ground state. The background would then have to be an unexciting background.

The simple harmonic oscillator (SHO) with time-dependent frequency is the simplest system where this question can be analyzed (see also Ref. [7]). Are there timedependent frequencies for which the SHO does not get excited? In this case we are able to find a complete solution in Sec. II. To our surprise, we find a very wide class of 


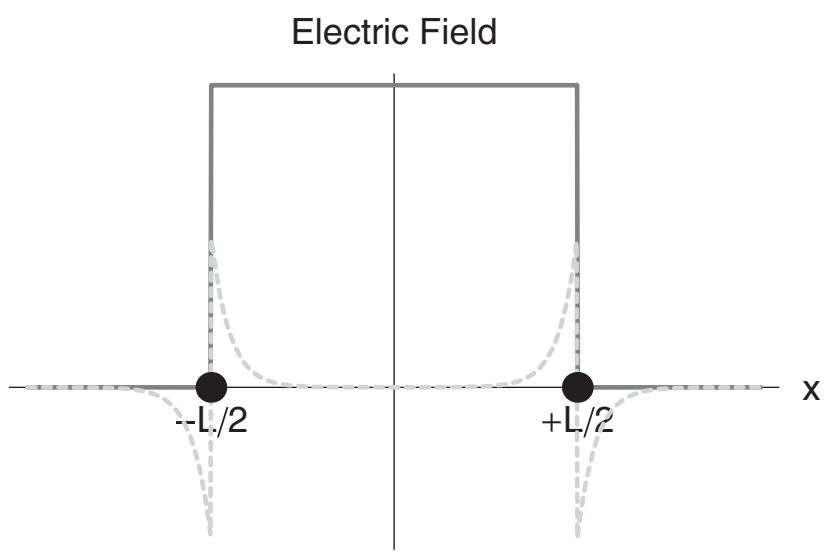

FIG. 1. As in Ref. [5], two infinitely heavy charges, $+Q$ and $-Q$, are placed at $x=-L / 2$ and $x=+L / 2$ respectively. The classical electric field is given by the thick dark line. With pair production, the electric field evolves into the unexciting configuration illustrated by the dashed curve.

time-dependent frequencies, not necessarily adiabatic, for which particle production does not occur.

The next step is to generalize the SHO result to quantum field theory in a classical background. We first consider homogeneous but time-dependent backgrounds. The homogeneity of the background simplifies matters since the excitations can be diagonalized and each mode of the quantum field behaves as a quantum SHO with timedependent frequency. In Sec. III, we show that there are backgrounds for which particle production can be suppressed for at most a discrete set of modes and not for all modes.

In Sec. IV we consider the full problem of inhomogeneous, time-dependent backgrounds. Here too we are able to find backgrounds for which there is no particle production. However, the solution does not address the constraint that only certain forms of interactions may be present in a particular physical system. After discussing whether field theory backgrounds may be unexciting at all times in Sec. VI, we turn to unexciting backgrounds that might arise in physical systems in Sec. VII. We are unable to construct a general unexciting physical background in a field theory and leave it as an open problem.

\section{QUANTUM SIMPLE HARMONIC OSCILLATOR}

Consider a SHO with unit mass $m=1$ and timedependent frequency $\omega(t)$. We are interested in finding $\omega(t)$ such that there is no net energy production in quantum excitations.

Our analysis uses the "classical-quantum correspondence" (CQC) developed in Refs. [8,9] whereby quantum particle production in time-dependent backgrounds can be analyzed by solving a system of classical differential equations in higher dimensions. (The formalism only applies to bosonic particles.) In the simplest case of a quantum SHO, the CQC maps the problem to a classical SHO in two dimensions, which can be described by a complex variable $z(t)$. Expectation values of quantum operators can all be written as functions of $z$.

\section{A. SHO Solution}

The CQC equation for the complex variable $z(t)$ is

$$
z^{\prime \prime}+\omega^{2} z=0
$$

with initial conditions (taken at $t=t_{i}$ )

$$
z_{i}=-\frac{i}{\sqrt{2 \omega_{i}}}, \quad z_{i}^{\prime}=-\sqrt{\frac{\omega_{i}}{2}}
$$

where primes denote time derivatives and subscripts $i$ and $f$ refer to initial and final times. The energy in excitations is given by the function ${ }^{1}$

$$
E(t)=\frac{1}{2}\left|z^{\prime}-i \omega z\right|^{2} .
$$

An unexciting background would be one for which the final energy in excitations vanishes. Note that excitations may be produced and absorbed at intermediate times; we only require the final energy to vanish for the background to be unexciting. If instead, we require that the energy vanishes at all times, (6) implies $z^{\prime}=i \omega z$. Differentiating once and using (4) implies $\omega^{\prime}=0$. Hence there are no nontrivial backgrounds for which the SHO excitation energy vanishes for all times.

To derive an unexciting background we first write

$$
z(t)=\rho(t) e^{i \theta(t)}
$$

Then (4) implies

$$
\rho^{\prime \prime}+\omega^{2} \rho=\frac{1}{4 \rho^{3}}, \quad \theta^{\prime}=-\frac{1}{2 \rho^{2}}
$$

where in the second equation we have used the initial conditions (5) in terms of $\rho$ and $\theta$,

$$
\rho_{i}=\frac{1}{\sqrt{2 \omega_{i}}}, \quad \rho_{i}^{\prime}=0, \quad \theta_{i}=\frac{3 \pi}{2}, \quad \theta_{i}^{\prime}=-\omega_{i} .
$$

Now we use the $\rho$ equation in (8) to solve for $\omega$ in terms of $\rho$,

\footnotetext{
${ }^{1}$ There is no ambiguity in the definition of excitation energy in contrast to the number of particles as discussed in the literature (e.g., [10] and, more recently, [11]).
} 


$$
\omega=\sqrt{\frac{1}{4 \rho^{4}}-\frac{\rho^{\prime \prime}}{\rho} .}
$$

This tells us how the frequency should vary with time for any choice of $\rho(t) \geq 0$. In addition, if we require $\omega^{2} \geq 0$, then $4 \rho^{3} \rho^{\prime \prime} \leq 1$, though $\omega^{2}<0$ implies an inverted SHO potential and might be acceptable for certain systems.

Now restrict the function $\rho(t)$ so that

$$
\rho_{i}^{\prime}=\rho_{i}^{\prime \prime}=0, \quad \rho_{f}^{\prime}=\rho_{f}^{\prime \prime}=0,
$$

while $\rho_{i}$ and $\rho_{f}$ are unconstrained. For any choice of such $\rho(t)$, the energy in excitations

$$
E(t)=\frac{\rho^{\prime 2}}{2}+\frac{\rho^{2}}{2}\left(\frac{1}{2 \rho^{2}}-\omega\right)^{2}
$$

satisfies

$$
E_{i}=0=E_{f}
$$

To see $E_{i}=0$, the initial conditions in (9) suffice. To see $E_{f}=0$, note that the function $\rho$ is chosen to satisfy $\rho_{f}^{\prime}=0$, so the first term in (12) vanishes, while $\rho_{f}^{\prime \prime}=0$ together with (10) implies that the second term in (12) vanishes at the final time. Note that $\rho_{i}$ and $\rho_{f}$ can be different, which means that $\omega_{i}$ and $\omega_{f}$ can be different.

In Fig. 2 we sketch the late time dynamics required for particle production.

Another quantity of interest may be the phase of the wave function, especially in cases where the final frequency equals the initial frequency. The full wave function

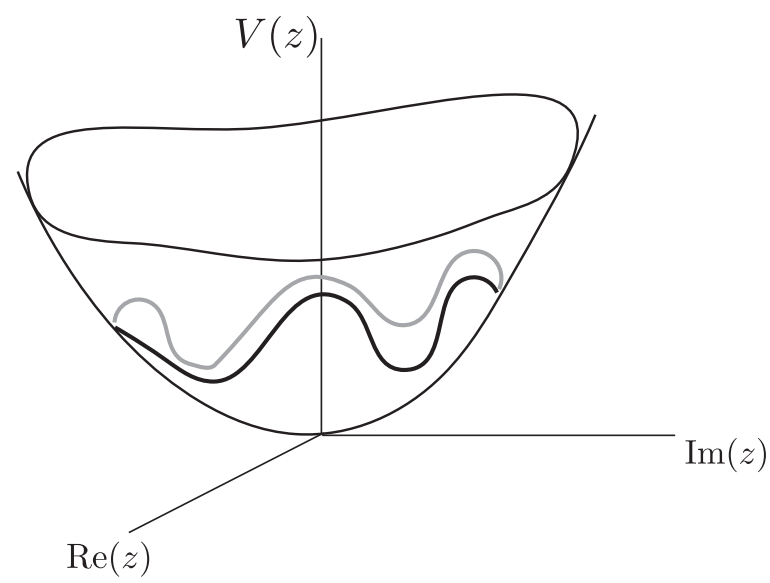

FIG. 2. The potential for the complex variable $z$ is parabolic in two dimensions (one complex dimension). Conservation of angular momentum implies that the trajectory of $z$ goes around the parabola. If the trajectory oscillates, as shown by the solid curve, there is net particle production. There is no net particle production if the trajectory does not oscillate at late times. for the position $x$ of the simple harmonic oscillator can be written as

$$
\psi(t, x)=\frac{e^{i \gamma(t)}}{\left(2 \pi \rho^{2}\right)^{1 / 4}} \exp \left[\frac{i}{2}\left(\frac{\dot{\rho}}{\rho}+\frac{i}{2 \rho^{2}}\right) x^{2}\right]
$$

where

$$
\gamma(t)=-\int_{t_{i}}^{t} \frac{d t^{\prime}}{4 \rho^{2}\left(t^{\prime}\right)}
$$

Let us now consider the case of an unexciting background with $\omega_{i}=\omega_{f}$. Then the conditions in (11) imply that $\rho_{i}=\rho_{f}$, and the phase difference from the case of a trivial background with $\omega(t)=\omega_{i}$ is

$$
\Delta \gamma(t)=-\frac{1}{4} \int_{t_{i}}^{t} d t^{\prime}\left(\frac{1}{\rho^{2}\left(t^{\prime}\right)}-\frac{1}{\rho_{i}^{2}}\right)
$$

\section{B. An explicit example}

Consider the choice of function

$$
\rho(t)=1+\frac{1}{2} \tanh (t),
$$

with $t_{i} \rightarrow-\infty$ and $t_{f} \rightarrow+\infty$. This choice satisfies the conditions $\rho_{i}^{\prime}=\rho_{i}^{\prime \prime}=0$ and $\rho_{f}^{\prime}=\rho_{f}^{\prime \prime}=0$ required for an unexciting background. Then (10) gives us $\omega(t)$ which we plot in Fig. 3 and in Fig. 4 we plot the energy in excitations as a function of time. At early times the energy in excitations grows but all the energy is absorbed at late times to give no net production of energy.

\section{A more general derivation}

The construction of the unexciting background in Sec. II A was explicit but it used polar coordinates that

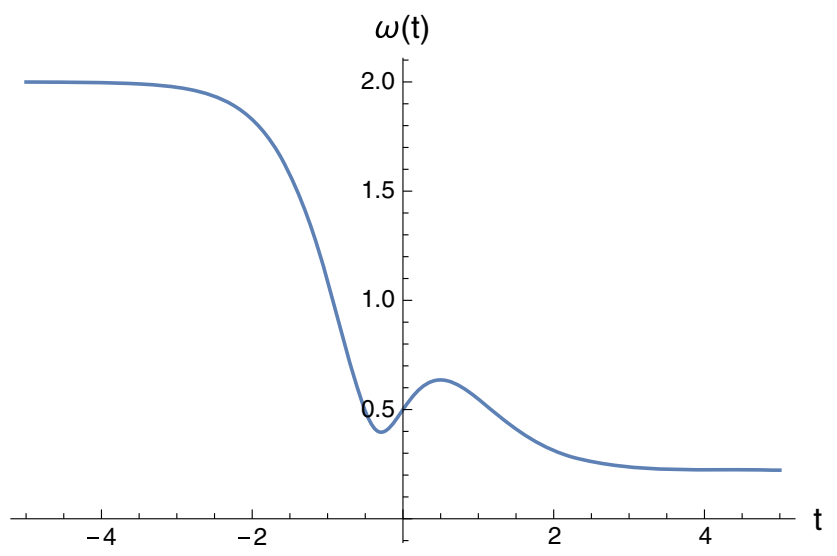

FIG. 3. The frequency $\omega(t)$ for the explicit example of Sec. II B. 


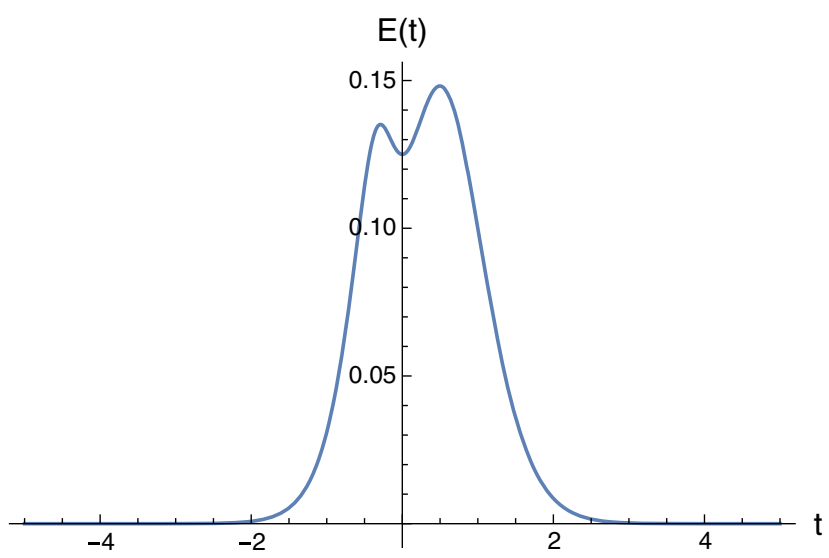

FIG. 4. The excitation energy $E(t)$ for the explicit example of Sec. II B.

do not generalize easily to the field theory case. Here we construct $\omega(t)$ in terms of the complex variables $z(t)$ and the procedure can be generalized to field theory as in Sec. IV.

We start with the identities

$\left(z z^{*}\right)^{\prime}=z z^{\prime *}+z^{\prime} z^{*}, \quad\left(z z^{*}\right)^{\prime \prime}=2\left(z^{\prime} z^{* \prime}-\omega^{2} z z^{*}\right)$

Therefore,

$F(t) \equiv\left(z^{\prime}-i \omega z\right)\left(z^{\prime}+i \omega z\right)^{*}=\frac{1}{2}\left(z z^{*}\right)^{\prime \prime}-i \omega\left(z z^{*}\right)^{\prime}$.

Now consider $z(t)$ such that

$$
\left(z z^{*}\right)_{i}^{\prime \prime}=0=\left(z z^{*}\right)_{i}^{\prime}
$$

and

$$
\left(z z^{*}\right)_{f}^{\prime \prime}=0=\left(z z^{*}\right)_{f}^{\prime}
$$

Then (19) shows that $F_{i}=0=F_{f}$, implying that one of the two factors $\left(z^{\prime}-i \omega z\right)$ or $\left(z^{\prime}+i \omega z\right)$ must vanish at $t_{i}$ and $t_{f}$. At $t_{i}$ the initial conditions tell us that

$$
\left(z^{\prime}-i \omega z\right)_{i}=0
$$

At $t_{f}$ we use the angular momentum constraint,

$$
z^{\prime} z^{*}-z z^{* \prime}=i
$$

and $\omega>0$ to show that

$$
\left|z^{\prime}+i \omega z\right|^{2}=\left|z^{\prime}\right|^{2}+\omega^{2}|z|^{2}+\omega>0
$$

provided $\omega>0$. Therefore $\left(z^{\prime}+i \omega z\right)_{f} \neq 0$. Then the only possibility is that

$$
\left(z^{\prime}-i \omega z\right)_{f}=0
$$

However the energy in excitations is given by (6) and hence $E_{f}=0$ if we have a $z(t)$ such that (21) is satisfied. With such a choice of $z(t)$ we find $\omega$ as

$$
\omega(t)=+\sqrt{-\frac{1}{2}\left(\frac{z^{\prime \prime}}{z}+\frac{z^{* \prime \prime}}{z^{*}}\right)}
$$

where we have made sure that the expression under the radical is real and we have only chosen the positive square root. With a little algebra, and making use of (23), we recover (10).

If we also require $\omega$ to be real valued, we must impose the condition

$$
-\frac{1}{2}\left(\frac{z^{\prime \prime}}{z}+\frac{z^{* \prime \prime}}{z^{*}}\right) \geq 0
$$

To summarize, an unexciting background can be found from

$$
\omega(t)=+\sqrt{-\frac{1}{2}\left(\frac{z^{\prime \prime}}{z}+\frac{z^{* \prime \prime}}{z^{*}}\right)}
$$

by choosing any complex function $z(t)$ that satisfies the conditions (20) and (21) together with the initial condition in (5) and the Wronskian condition in (23).

The solution in (31) is equivalent to the solution in (10) when written in terms of $\rho$ and $\theta$ together with the constraint in (23).

\section{HOMOGENEOUS BACKGROUNDS}

If the background is time dependent but spatially homogeneous, the quantum field can be expanded in Fourier modes and the problem reduces to an infinite number of simple harmonic oscillators labeled by the wave number of that mode. The time-dependent frequency of each mode is denoted $\omega_{\mathbf{k}}(t)$ and depends on the background under consideration. The variables corresponding to the $z$ 's for the single harmonic oscillator of Sec. II now carry the mode index and will be written as $z_{\mathbf{k}}$. They satisfy the equation

$$
z_{\mathbf{k}}^{\prime \prime}+\omega_{\mathbf{k}}^{2} z_{\mathbf{k}}=0
$$

with initial conditions in (5). The frequencies $\omega_{\mathbf{k}}$ may take different forms depending on the interactions in question. We will illustrate the arguments for the form when a classical background field, $\phi(t)$, interacts with a quantum field, $\psi(\mathbf{x}, t)$, due to a $\lambda \phi^{2} \psi^{2} / 2$ interaction. Then, 


$$
\omega_{\mathbf{k}}^{2}=\mathbf{k}^{2}+\lambda \phi^{2}(t) .
$$

From Sec. II we can certainly find a background for which a given mode is not excited. But we are interested in finding a background for which none of the modes is excited. Let us choose a background for which the mode $\mathbf{k}=\mathbf{k}_{*}$ is unexcited and denote the mode by $*$ subscripts. Then the background is given by

$$
\omega_{*}^{2}(t)=-\frac{1}{2}\left(\frac{z_{*}^{\prime \prime}}{z_{*}}+\frac{z_{*}^{* \prime \prime}}{z_{*}^{*}}\right)
$$

and

$$
\lambda \phi^{2}(t)=-\mathbf{k}_{*}^{2}-\frac{\rho_{*}^{\prime \prime}}{\rho_{*}}+\frac{1}{4 \rho_{*}^{4}} .
$$

Then, for another mode, say $\mathbf{k}=\mathbf{p}$, we must have

$$
z_{\mathbf{p}}^{\prime \prime}+\left[\left(\mathbf{p}^{2}-\mathbf{k}_{*}^{2}\right)+\omega_{*}^{2}(t)\right] z_{\mathbf{p}}=0
$$

and initial conditions for $z_{\mathbf{p}}$ are as in (5). For $z_{\mathbf{p}}$ to be unexcited, we require that $z_{\mathbf{p}}^{\prime \prime}\left(t_{f}\right)=0$. However, the initial conditions fix the evolution of $z_{\mathbf{p}}$ and the condition $z_{\mathbf{p}}^{\prime \prime}\left(t_{f}\right)=0$ is an extra boundary condition on the evolution. In general, it will only be satisfied for at most a discrete set of modes, not for all p. Hence we conclude that unexciting homogeneous backgrounds do not exist. (Time-dependent electric fields with no Schwinger pair production in a particular mode are discussed in [12].)

The story would be different if each mode of the quantum field were to interact with an independent background. Then one would be able to separately choose unexciting backgrounds for each mode. This suggests that perhaps inhomogeneous backgrounds, where different background modes couple to different excitation modes, can be unexciting. We now turn to this question.

\section{GENERAL SPACE AND TIME-DEPENDENT BACKGROUNDS}

A free quantum field in a general space and timedependent background can be treated within the framework of the CQC. Then space is discretized, say with $N$ lattice points, and the Bogolyubov coefficients (generalized to inhomogeneous backgrounds) correspond to an $N \times N$ matrix that we denote by $Z$. The equation of motion for $Z$ is

$$
Z^{\prime \prime}+\Omega^{2} Z=0
$$

where $\Omega=\Omega^{\dagger}=\Omega^{*}$ contains both the spatial derivatives of the (real) field and the spacetime background.
The initial conditions for $Z$ are ${ }^{2}$

$$
Z_{i}=-\frac{i}{\sqrt{2}}\left(\sqrt{\Omega_{i}}\right)^{-1}, \quad Z_{i}^{\prime}=\frac{1}{\sqrt{2}} \sqrt{\Omega_{i}}
$$

where it is assumed that $\Omega_{i}$ is invertible.

The matrix $\Omega^{2}$ is a combination of the gradient terms for the quantum field and its interactions with the background. Hence we write

$$
\Omega^{2}=-\nabla^{2}+U
$$

where

$$
\nabla^{2}= \begin{cases}-2 / a^{2}, & i=j \\ 1 / a^{2}, & i=j \pm 1 \\ 0, & \text { otherwise }\end{cases}
$$

where $a$ is the lattice spacing. The form of the matrix $U$ is constrained by the form of the interactions. For example, if the interactions are local, i.e., occur at the same spatial point, then $U$ will be diagonal. For derivative interactions, $U$ will contain off-diagonal terms.

Further we have constraints that are satisfied by the evolution [9],

$$
\begin{gathered}
Z^{* \prime} Z^{T \prime}-Z^{\prime} Z^{\dagger \prime}=0 \\
Z^{*} Z^{T}-Z Z^{\dagger}=0 \\
Z^{*} Z^{T \prime}-Z Z^{\dagger \prime}=i .
\end{gathered}
$$

These constraints can also be recast as [9]

$$
\begin{gathered}
Z^{\dagger} Z^{\prime}-Z^{\dagger \prime} Z=i \\
Z^{\dagger} Z^{* \prime}-Z^{\dagger \prime} Z^{*}=0 .
\end{gathered}
$$

The total energy in quantum excitations is given by

$$
E=\frac{1}{2} \operatorname{Tr}\left|Z^{\prime}-i \Omega Z\right|^{2}
$$

and we define an unexciting background to be one that gives $E_{f}=0$.

First we derive a necessary condition for an unexciting background. From (43), $E\left(t_{f}\right)=0$ implies

$$
Z_{f}^{\prime}=i \Omega_{f} Z_{f}, \quad Z_{f}^{\dagger \prime}=-i Z_{f}^{\dagger} \Omega_{f} .
$$

Multiplying these two equations and simplifying gives

\footnotetext{
${ }^{2}$ To take the positive square root, the matrix under the radical is diagonalized, then the positive square root of each of the diagonal entries is taken, and finally the matrix diagonalization is inverted.
} 


$$
\left(Z^{\dagger} Z\right)_{f}^{\prime \prime}=0 .
$$

Equation (45) is a necessary condition to construct an unexciting background. Once we find a suitable $Z$, the unexciting background is given by

$$
\Omega^{2}=-\frac{1}{2}\left(Z^{\prime \prime} Z^{-1}+\left(Z^{\dagger}\right)^{-1} Z^{\dagger \prime}\right)
$$

and

$$
\Omega=+\sqrt{-\frac{1}{2}\left(Z^{\prime \prime} Z^{-1}+\left(Z^{\dagger}\right)^{-1} Z^{\dagger \prime \prime}\right)}
$$

where the + sign indicates that the positive (matrix) root should be taken.

The condition (45), together with the constraints in (38), (39) and (40), and the additional condition

$$
\left(Z^{\dagger} Z\right)_{f}^{\prime}=0
$$

are also sufficient for an unexciting background. To show this, we rewrite (46) as

$$
\Omega^{2}=-\frac{1}{2}\left(Z^{\dagger}\right)^{-1}\left(\left(Z^{\dagger} Z\right)^{\prime \prime}-2 Z^{\dagger \prime} Z^{\prime}\right) Z^{-1} .
$$

Having chosen some $Z(t)$, (49) fixes $\Omega^{2}$ for all times.

We now show that (47) gives vanishing energy at the final time. This is because the condition in (45) when inserted in (49) gives

$$
\Omega_{f}=+\sqrt{\left(-i Z_{f}^{\prime} Z_{f}^{-1}\right)^{\dagger}\left(-i Z_{f}^{\prime} Z_{f}^{-1}\right)} .
$$

Next we show that $M \equiv-i Z_{f}^{\prime} Z_{f}^{-1}$ is Hermitian.

$$
M-M^{\dagger}=-i\left(Z_{f}^{\dagger}\right)^{-1}\left(Z^{\dagger} Z\right)_{f}^{\prime} Z_{f}^{-1}=0,
$$

since $Z(t)$ is chosen to satisfy (48). Therefore $M=M^{\dagger}$. Further, using the constraint in (41),

$$
M=\frac{1}{2}\left(M+M^{\dagger}\right)=\left(Z Z^{\dagger}\right)_{f}^{-1}
$$

and this is a positive matrix. Therefore (50) gives

$$
\Omega_{f}=-i Z_{f}^{\prime} Z_{f}^{-1}=\left(Z Z^{\dagger}\right)_{f}^{-1}
$$

and so from (43),

$$
E_{f}=\frac{1}{2} \operatorname{Tr}\left|Z_{f}^{\prime}-i \Omega_{f} Z_{f}\right|^{2}=0 .
$$

This proves that to construct an unexciting background we can use (47) where $Z(t)$ satisfies the constraints in (38), (39) and (40), and the final time conditions in (45) and (48).

\section{SOLVING THE CONSTRAINTS}

Let us define

$$
\rho^{2}=Z Z^{\dagger}
$$

where $\rho^{2}$ is real, symmetric and positive due to the constraint condition in (39). Then we can write

$$
Z=\rho U
$$

where $U$ is a unitary matrix.

Now we turn to the constraint in (40). Insertion of (56) in (40) gives the conditions

$$
\begin{gathered}
{\left[\rho, \rho^{\prime}\right]=0,} \\
\left\{\rho^{2}, U^{\prime} U^{\dagger}\right\}=i,
\end{gathered}
$$

where the curly braces denote an anticommutator. Note that (57) also implies $\left[\rho, \rho^{\prime \prime}\right]=0$.

A solution of Eq. (58) is

$$
U^{\prime} U^{\dagger}=\frac{i}{2} \rho^{-2}
$$

which is analogous to the solution in the case of a single simple harmonic oscillator [see Eq. (8)]. ${ }^{3}$

With some algebra, we can check that $Z$ as given by (56) satisfies all the three constraints (38), (39) and (40). With this $Z$ in (46) we also find

$$
\Omega^{2}=-\rho^{\prime \prime} \rho^{-1}+\frac{1}{4} \rho^{-4} .
$$

So now the problem of constructing field theory unexciting backgrounds has been reduced to suitably choosing a real-valued matrix function $\rho$ that satisfies the conditions

$$
\left[\rho, \rho^{\prime}\right]=0, \quad \rho_{i}^{\prime}=0=\rho_{f}^{\prime}, \quad \rho_{i}^{\prime \prime}=0=\rho_{f}^{\prime \prime} .
$$

Then we can construct $\Omega^{2}$ using (60).

A simple example solution is

$$
\rho(t)=A+\frac{1}{2} \tanh (t) B
$$

where the time-independent, real, symmetric matrices $A$ and $B$ commute: $[A, B]=0$. This choice of $\rho$ satisfies all the conditions in (61) and from (60) will lead to $\Omega^{2}$ that is unexciting. The challenge however is to find $\rho(t)$ that not only gives an unexciting background but is also consistent

\footnotetext{
${ }^{3}$ The solution in (59) is not unique. For example, one could add any matrix on the right-hand side of (59) that anticommutes with $\rho^{2}$.
} 
with interactions that are of physical interest. We will turn to this question in Sec. VII.

\section{UNEXCITING FOR ALL TIMES}

In the case of the SHO it was simple to see that only the trivial background with $\omega^{\prime}=0$ is unexciting at all times as in Sec. II A. Here we consider field theory backgrounds that may be unexciting for all times.

Setting $E(t)=0$ in (43) gives

$$
Z^{\prime}=i \Omega Z \text {. }
$$

Differentiating once with respect to time and using (34) gives

$$
\Omega^{\prime} Z=0 .
$$

Assuming that $Z$ is invertible, this implies that $\Omega^{\prime}=0$ and the time dependence of $\Omega$ is trivial. An exception is when the background has some symmetries and there are excitation zero modes for then the initial $Z$ in (35) is not well defined.

A second related exception is in situations where the background is "stationary." Then the background can have time dependence but the spectrum of excitations is time independent and so $\Omega^{\prime}=0$. An example is when the background is due to a soliton, as discussed in [13]. A static soliton background has translational symmetry and the excitation spectrum has a zero mode [14]. A boosted soliton background is time dependent but there is clearly no particle production since one can always go to the rest frame of the soliton. A second similar example of an unexciting background is that of pp waves [15] and may be relevant for cosmologies with null big bang singularities [16]. A third example occurs in spontaneously broken nonAbelian gauge theories that contain monopole solutions. Excitations of the rotor degree of freedom of the monopole endows the monopole with electric charge and converts it into a dyon [17]. The dyon fields are time dependent but stationary, and the spectrum of excitations around a dyon is time independent. This then brings us to the example of a pure non-Abelian gauge theory. Here too there are rotor degrees of freedom whose time dependence produces stationary backgrounds [18]. We plan to describe and analyze this example in a forthcoming publication.

Another important point to note is in the context of massless QED in $1+1$ dimensions mentioned in the Introduction. There we have described an unexciting electric field configuration. This background is unexciting for all times as no fermions are produced, in contrast to our conclusion above. The reason is that our analysis using the CQC only applies to the production of bosons and cannot be applied to fermionic systems. Once the model is bosonized, the scalar field, $\phi$, couples directly to the electromagnetic field strength due to a $\phi \epsilon^{\mu \nu} F_{\mu \nu}$ coupling.
Even though the gauge potential is time dependent in temporal gauge, there can be no production of $\phi$ quanta in a static electric field background.

\section{UNEXCITING PHYSICAL BACKGROUNDS?}

The system of interest may be a quantum field interacting with a scalar background, for example a $\lambda \phi^{2} \psi^{2} / 2$ interaction as in Sec. III. Or it could be charged particles interacting with a background electric field, as in Schwinger particle production. Or it could be both a scalar field and an electric field, and also perhaps a gravitational background. Depending on the system, the form of the frequency matrix $\Omega^{2}$ is restricted and it is of interest to find unexciting backgrounds consistent with the interactions of interest.

Let us illustrate the problem with our example from Sec. III where the interaction is $\lambda \phi^{2} \psi^{2} / 2$ and $\phi(t, \mathbf{x})$ is the space- and time-dependent background. In this case the interaction acts like an effective mass term and $\Omega^{2}$ takes the form,

$$
\Omega^{2}=-\nabla^{2}+\left(m^{2}+\lambda \phi^{2}\right)
$$

where $\nabla^{2}$ is given in (37) and is a symmetric, tri-diagonal matrix, while the $m^{2}+\lambda \phi^{2}$ term is a diagonal matrix. From (60) we can write

$$
\lambda \phi^{2}=-\left(\square \rho+m^{2} \rho-\frac{1}{4} \rho^{-3}\right) \frac{1}{\rho}
$$

where $\square=\partial_{t}^{2}-\nabla^{2}$ is the D'Alembertian (matrix) operator. Since $\lambda \phi^{2}$ has to be a diagonal matrix, this imposes an additional constraint on $\rho$, namely that the right-hand side of (66) be diagonal. It is not clear how to choose a nontrivial $\rho(t)$ that satisfies (61) and that leads to a diagonal form for $\lambda \phi^{2}$ in (66).

The physical system of quantum excitations in a color electric field [4] is similar to that of the scalar field discussed above but with additional complications due to group indices and three spatial dimensions. The background vector gauge potential can be taken in temporal gauge to be $A_{i}^{a}=E_{i}(\mathbf{x}) f(t) \delta^{a 3}$, where $E_{i}(\mathbf{x})$ is the chosen background electric field function of the $a=3$ color, and the function $f(t)$ is chosen to suitably turn the electric field on and off asymptotically. (We assume that external currents are present so that the background magnetic field vanishes.) The leading interaction between the background and the gluonic excitations will again be local; only the gradient terms provide couplings of the excitation fields at different spatial points. The analog of (66) for this problem will again require that a matrix $\rho$ be chosen so that a combination similar to that on the right-hand side of (66) be diagonal. 


\section{CONCLUSIONS}

Our investigations were motivated by Schwinger pair production in the background of a non-Abelian electric field, but the question is more general-are there classical time-dependent backgrounds that do not produce quantum excitations?

To address this question, we first considered a quantum simple harmonic oscillator with a time-dependent frequency. We found an infinite set of unexciting backgrounds-variations of the frequency, even possibly rapid, that lead to no net production of excitations. The result is potentially of interest in practical settings where one may wish to alter external backgrounds without disturbing a quantum system.

We then considered the quantum field theory case. The spatially homogeneous background problem can be diagonalized and becomes equivalent to an infinite set of simple harmonic oscillators. We argued that we could suppress excitations of some modes by choosing a suitable background time dependence. However, there are always some modes that get excited by the time-dependent background and hence a homogeneous background cannot be unexciting.

Finally we considered the general case of inhomogeneous, time-dependent backgrounds. Here we were able to derive a formula that enables us to construct unexciting backgrounds. However these are "idealized" backgrounds and, as discussed in Sec. VII, may not correspond to physical interactions, e.g., an electric field background. The question whether there are unexciting physical backgrounds is still open, one we hope to return to in the future.

Another question of interest that we considered in Sec. VI is if there are classical time-dependent backgrounds that are unexciting for all times. We showed that such backgrounds may exist in bosonic systems provided the background has symmetries and the time dependence is purely in the variables that are conjugate to the symmetry generators. For then, the time dependence leads to a stationary background in which the spectrum of excitations is time independent and hence there is no particle production. A related question is to find backgrounds in which excitations are continuously created and absorbed in an oscillatory fashion, with no net production on average.

\section{ACKNOWLEDGMENTS}

I thank George Zahariade for many helpful discussions and Carlos Cardona, Sang Pyo Kim, Mainak Mukhopadhyay, Christian Schubert and Savdeep Sethi for comments. This work was supported by the U.S. Department of Energy, Office of High Energy Physics, under Award No. DE-SC0019470 at ASU.
[1] N. D. Birrell and P. C. W. Davies, Quantum Fields in Curved Space, Cambridge Monographs on Mathematical Physics (Cambridge University Press, Cambridge, England, 1984), ISBN 978-0-521-27858-4, 978-0-521-27858-4.

[2] J. S. Schwinger, Phys. Rev. 82, 664 (1951).

[3] S. W. Hawking, Commun. Math. Phys. 43, 199 (1975); 46, 206(E) (1976).

[4] C. Cardona and T. Vachaspati, Phys. Rev. D 104, 045009 (2021).

[5] Y.-Z. Chu and T. Vachaspati, Phys. Rev. D 81, 085020 (2010).

[6] G. Gold, D. A. Mcgady, S. P. Patil, and V. Vardanyan, J. High Energy Phys. 10 (2021) 072.

[7] S. P. Kim, New Phys. 71, 1082 (2021).

[8] T. Vachaspati and G. Zahariade, Phys. Rev. D 98, 065002 (2018).

[9] T. Vachaspati and G. Zahariade, J. Cosmol. Astropart. Phys. 09 (2019) 015.

[10] R. Dabrowski and G. V. Dunne, Phys. Rev. D 94, 065005 (2016).
[11] A. Ilderton, Phys. Rev. D 105, 016021 (2022).

[12] S. P. Kim and C. Schubert, Phys. Rev. D 84, 125028 (2011).

[13] M. Mukhopadhyay, E. I. Sfakianakis, T. Vachaspati, and G. Zahariade, arXiv:2110.08277.

[14] T. Vachaspati, Kinks and Domain Walls: An Introduction to Classical and Quantum Solitons (Cambridge University Press, Cambridge, England, 2010), ISBN 978-0-521-141918, 978-0-521-83605-0, 978-0-511-24290-8.

[15] S. Deser, J. Phys. A 8, 1972 (1975).

[16] B. Craps, S. Sethi, and E. P. Verlinde, J. High Energy Phys. 10 (2005) 005.

[17] S. R. Coleman, The magnetic monopole fifty years later, in The Unity of the Fundamental Interactions, edited by A. Zichichi (Springer, Boston, MA, 1983), pp. 21-117, 10.1007/978-1-4613-3655-6_2.

[18] L. S. Brown and W. I. Weisberger, Nucl. Phys. B157, 285 (1979); B172, 544(E) (1980). 